Article

\title{
Settlement Prediction of Footings Using $V_{S}$
}

\author{
Hyung Ik CHO ${ }^{1}$, Nam Ryong KIM ${ }^{2}$, Heon Joon PARK ${ }^{3}$ (D) and Dong Soo KIM ${ }^{3, *}$ \\ 1 Earthquake Research Center, Korea Institute of Geoscience and Mineral Resources (KIGAM), \\ Daejeon 34132, Korea; hicho@kigam.re.kr \\ 2 Infra Safety Research Center, K-water Institute, Daejeon 34045, Korea; namryong@kwater.or.kr \\ 3 Department of Civil and Environmental Engineering, Korea Advanced Institute Science and \\ Technology (KAIST), Daejeon 34141, Korea; heonjoon@kaist.ac.kr \\ * Correspondence: dskim@kaist.ac.kr; Tel.: +82-42-350-3619
}

Received: 29 September 2017; Accepted: 23 October 2017; Published: 25 October 2017

\begin{abstract}
The shear wave velocity $\left(V_{S}\right)$ is a key parameter for estimating the deformation characteristics of soil. In order to predict the settlement of shallow footings in granular soil, the $V_{S}$ and the concept of Schmertmann's framework were adopted. The $V_{S}$ was utilized to represent soil stiffness instead of cone tip resistance $\left(q_{c}\right)$ because the $V_{S}$ can be directly related to the small-strain shear modulus. By combining the $V_{S}$ measured in the field and the modulus reduction curve measured in the laboratory, the deformation characteristics of soil can be reliably estimated. Vertical stress increments were determined using two different profiles of the strain influence factor $\left(I_{z}\right)$ proposed in Schmertmann's method and that calculated from the theory of elasticity. The corresponding modulus variation was determined by considering the stress level and strain at each depth. This state-dependent stress-strain relationship was utilized to calculate the settlement of footings based on the theory of elasticity. To verify the developed method, geotechnical centrifuge tests were carried out. The $V_{S}$ profiles were measured before each loading test, and the load-settlement curves were obtained during the tests. Comparisons between the measured and estimated load-settlement curves showed that the developed method adequately predicts the settlement of footings, especially for over-consolidated ground conditions.
\end{abstract}

Keywords: settlement; shallow footing; shear wave velocity; centrifuge test

\section{Introduction}

The design paradigm of civil structures is currently shifting to performance-based design (PBD) instead of the traditional safety factor-based design. In PBD, greater emphasis is placed on controlling structural deformation to assure the serviceability and durability of civil structures [1] rather than using safety factors for design. Specifically, the key design factor of shallow foundation is settlement and not the bearing capacity because shallow foundations are generally constructed on good ground conditions having sufficient bearing capacity.

A number of methods have been introduced to predict the settlement of shallow foundations. Most of these methods are based on the theory of elasticity and thus focus on the determination of soil compressibility. In order to determine the compressibility of soil (i.e., stiffness or modulus) the selection of stiffness is commonly made on the basis of penetration resistance. Parameters such as the $N$ value and $q_{c}$ value, derived from a standard penetration test (SPT) and cone penetration test $(\mathrm{CPT})$, respectively, are generally utilized to determine the stiffness of soil layers. Meyerhof [2], Peck \& Bazaraa [3], and Burland \& Burbidge [4] suggested methods of settlement estimation for footings based on the SPT- $N$ value while Schmertmann [5], Schmertmann et al. [6] and Berardi et al. [7] suggested methods based on the CPT- $q_{c}$ value. 
Schmertmann's method has been extensively applied by engineers due to its simplicity and intuitive concept based on elasticity theory. The method utilizes CPT tip resistance, $q_{c}$, to evaluate Young's modulus using soil type-based empirical coefficients. For predicting settlement, the crucial factor is, however, deformation-related stiffness and not strength. Even though modulus determination from SPT- $N$ or CPT- $q_{c}$ is generally adopted, the penetration resistance is strongly related to the soil strength and requires additional empirical coefficients to convert soil strength to soil modulus. The coefficient utilized for conversion ranges from 2 to 24 depending on the soil condition [8]. Therefore, it is quite probable that the penetration resistance-based methods could estimate the settlement several times greater or smaller due to the wide range of coefficients depending on soil condition. This is the intrinsic limitation of using strength parameters in the settlement prediction. In addition, these in-situ tests are not applicable under certain ground conditions such as intermediate to hard geomaterials (e.g., weathered soil/rock).

As mentioned earlier, most of the penetration resistance-based methods adopt the elasticity theory. These methods, including Schmertmann's method, assume linear elastic soil deformation using a representative modulus taken from the resistance values. However, observations of settlement on footings and soil behavior show a high degree of nonlinearity, which changes with the stress-strain level. Previous studies have reported that settlement prediction based on nonlinear stress-strain models is reasonably consistent with field observations [9]. Recently, the nonlinearity of soil behavior was considered in settlement predictions according to PBD for enhancing the accuracy of settlement prediction. Lee et al. [10] and O'Loughlin \& Lehane [11] proposed a modified Schmertmann's method using modulus which varied depending on the settlement or strain level rather than using a single representative modulus initially converted from the $q_{c}$ value. Nevertheless, the penetration resistance based on strength criteria is still commonly utilized in modulus determination using relevant coefficients.

The shear wave velocity $\left(V_{S}\right)$ could be an alternative parameter in the settlement prediction to resolve the aforementioned problems. The $V_{S}$ is directly related to the deformation characteristics of soil (i.e., the maximum shear modulus, $G_{\max }$ ). Complete soil deformation characteristics could be established from the $V_{S}$. The maximum shear modulus $\left(G_{\max }\right)$ below the elastic threshold strain, which is the fundamental stiffness, is usually inferred from the $V_{S}$ obtained by seismic wave propagation tests. The variation in shear modulus with strain can be determined at small to intermediate strains by laboratory tests such as the resonant column test, torsional shear test and small-strain triaxial test. Finally, reliable nonlinear stiffness variation could be determined by combining the $G_{\max }$ and $G / G_{\max }$ curve, and hence the $V_{S}$, which is related to $G_{\max }$, can be used as a key soil property for the deformation analysis of soil-foundation systems [12]. Besides, the $V_{S}$ can be measured non-intrusively in the gravel and cobble layers, which are difficult to characterize using penetration testing methods. Indeed, the $V_{S}$ has great potential to replace the role of CPT- $q_{c}$ or SPT- $N$ values in the settlement prediction methods for determining the deformation modulus.

This study aims to develop a new approach for predicting the load-settlement behavior of shallow footings based on the $V_{S}$ and nonlinearity of soil. The development of a new method was based on the conceptual framework of Schmertmann's method [6], which is one of the commonly used methods in engineering practice. The procedures for obtaining confinement and strain dependent modulus values from the $V_{S}$ profile considering pressure distribution under the footing were developed. To verify the developed method, load-settlement curves for footings having a different length to breadth ratio, together with $V_{S}$ profiles, were obtained by centrifuge modeling, and then compared with the predictions from the developed method. 


\section{Development of Settlement Prediction Method Based on $V_{S}$}

\subsection{Schmertmann's Approach}

Here, we adopt the conceptual framework of Schmertmann's method to establish a new settlement prediction method. The settlement of footing as suggested by Schmertmann et al. [6] is expressed as:

$$
s_{i}=C_{1} \cdot C_{2}\left(q_{b}-\sigma_{z, d}^{\prime}\right) \sum \frac{I_{z i} \Delta z_{i}}{E_{i}}
$$

where $C_{1}$ and $C_{2}$ are depth and time factors, $q_{b}$ is the footing load, $\sigma_{z, d}^{\prime}$ is the initial vertical effective stress at the footing base level, $I_{z i}$ is the vertical strain influence factor, $\Delta z_{i}$ is the thickness of each sublayer, and $E_{i}$ is the elastic modulus of each layer.

Schmertmann's method utilizes the CPT tip resistance, $q_{c}$, to determine Young's modulus using soil type-based empirical coefficients. The stress transmitted into the soil media, which is caused by the footing load, can be calculated by using a strain influence factor, $I_{z}$, which describes the distribution of the applied load in the subsoil layer with respect to depth. Then, the settlement of each sublayer can be evaluated using the converted modulus and transmitted stress based on elasticity theory, and finally the total settlement of footing over the soil profile is obtained.

In this study, the use of $q_{c}$ in the determination of Young's modulus was replaced by the $V_{S}$ incorporating the nonlinearity of soil. Based on the $V_{S}$, the modulus was determined considering the confinement stress and strain level caused by pressure distribution under the footing. In addition, the shape of $I_{z}$ was discussed to provide reliable results using the developed method.

\subsection{Modulus Determination of the Soil Layer}

Many of the proposed stress-strain equations are limited in terms of the function of shear strain. In contrast, Fahey [13] reported that hyperbolic equations can be rewritten as a function of shear stress rather than shear strain to describe the nonlinear stress-stain relationship. Fahey \& Carter [14] proposed two additional model parameters $f$ and $g$ to have flexibility in changing the shape of the stress-strain curve and to adjust the model to approach failure at finite strain. The proposed equation is given as:

$$
\frac{G}{G_{0}}=1-f\left(\frac{\tau}{\tau_{\max }}\right)^{g}
$$

where $G$ is the secant shear modulus, $G_{0}$ is the small-strain shear modulus, $\tau$ is shear stress and $\tau_{\max }$ is the shear strength.

Previous studies show that this modified hyperbolic model can be used in load-settlement predictions, simple hand calculation [15], and finite element studies [9,16]. It is convenient to adopt this modified hyperbolic model for the estimating footing's settlement because the transmitted stress into the soil media caused by the footing load (or design load) can be derived by load transfer mechanisms, such as the strain influence factor or elastic theory using Boussinesq's equation. Thus, the modulus reduction can be considered as a function of the mobilized stress level (i.e., $G / G_{0}$ versus $\left.\tau / \tau_{\max }\right)$, and it is more advantageous than using strain.

While the footing is loaded, the confining stress increases in the underlying soil and this effect can be considered as a change in $G_{0}$ as:

$$
G_{o}\left(\sigma_{2}\right)=\left(\frac{\sigma_{2}}{\sigma_{1}}\right)^{n} G_{o}\left(\sigma_{1}\right)
$$

where $\sigma_{1}$ is the initial effective confining stress, and $\sigma_{2}$ is the effective confining stress with the footing load. The stress state-dependent shear modulus in Equation (3) can be used in the settlement prediction procedure to reflect a reasonable stress increment in the soil; this approach was validated from previous 
settlement predictions $[9,15,16]$. The initial $G_{0}$ can be directly calculated from $V_{S}$ and mass density $(\rho)$ according to the following equation:

$$
G_{o}=\rho V_{s}^{2}
$$

Assuming constant Poisson's ratio, $v$, regardless of strain level, the modified hyperbolic model in Equation (2) can be rewritten as the axial stress-stain formula:

$$
\frac{E}{E_{0}}=1-f\left(\frac{\Delta \sigma_{v}}{\sigma_{\max }}\right)^{g}
$$

where $E$ is secant Young's modulus, $E_{o}$ is small-strain Young's modulus, and $\Delta \sigma_{v}$ and $\sigma_{\max }$ are the vertical deviator stress and ultimate bearing capacity, respectively. The secant modulus, $E$, in Schmertmann's method can be evaluated by the small-strain shear modulus directly calculated from the $V_{S}$ and the nonlinear stress-strain equation. Combining the modulus reduction by the stress (or strain) level in Equation (5) with the increasing modulus by effective confining stress increment in Equation (3), the design modulus can be determined as:

$$
E=E_{o}\left(\frac{\sigma_{o v}+\Delta \sigma_{v}}{\sigma_{o v}}\right)^{n}\left(1-f\left(\frac{\Delta \sigma_{v}}{\sigma_{\max }}\right)^{g}\right)
$$

where $E_{o}$ is the initial small strain Young's modulus, $\sigma_{o v}$ is the theoretically calculated vertical effective stress and $\Delta \sigma_{v}$ is the vertical stress increment by footing load. Based on Equation (6), the stiffness of soil layers can be calculated considering both the modulus increment by increasing effective confining stress and modulus reduction by stress or strain level using $V_{S}$ measurements directly.

\subsection{Stress Distribution Beneath Footings}

As mentioned earlier, the stress transmitted into the soil media caused by the footing load can be derived from load transfer mechanisms, such as the strain influence factor $\left(I_{z}\right)$ or elasticity theory using Boussinesq's equation. Figure 1 shows $I_{z}$ profiles for square footing (length to breadth ratio, $L / B=1.0)$ and strip footing $(L / B \geq 10.0)$. Schmertmann et al. [6] proposed a simple triangular $I_{z}$ with a peak value occurring at $0.5 B$ depth, and the value converges to zero at $2.0 B$ depth for square footing, while the peak and zero values appear at $1.0 \mathrm{~B}$ and $4.0 \mathrm{~B}$ depth, respectively, for strip footing. Further, in Schmertmann's $I_{z}$ profile, the peak value increases with increasing applied pressure. This is used when considering the nonlinear characteristics of soil because the fixed modulus calculated from $\mathrm{CPT}-q_{c}$ does not consider the modulus degradation with respect to stress (or strain) level. Without changing the modulus, Schmertmann et al. [6] intended to increase the applied stress within a soil mass to take into account the nonlinear deformation behavior by increasing the peak value of the $I_{z}$.

In the developed method, secant Young's modulus changes based on the nonlinear property of soil, which depends on the initial stress state (i.e., $V_{S}$ ) and design load. On the contrary, in Schmertmann's method, a fixed modulus converted from CPT- $q_{c}$ is utilized while the nonlinear soil property is considered by the variation of $I_{z}$. Thus, in the newly developed method, the $I_{z}$ should be invariable regardless of the design load so that the nonlinear property of soil is not considered twice in the modulus determination. In fact, Schmertmann's triangles were proposed for convenience in the application, and their simple shape was determined to accord with the peak value changing with the design load. Although Schmertmann's triangle has an advantage in engineering practice due to its simplicity, definite error has been reported because of the simplicity. Mayne \& Poulos [17] reported that clipping the $I_{z}$ arbitrarily below $z=2 \mathrm{~B}$ for square footing results in $18 \%$ unconservative error. 


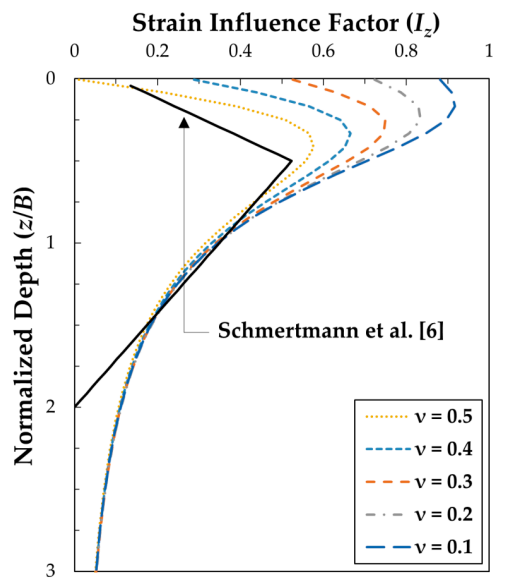

(a)

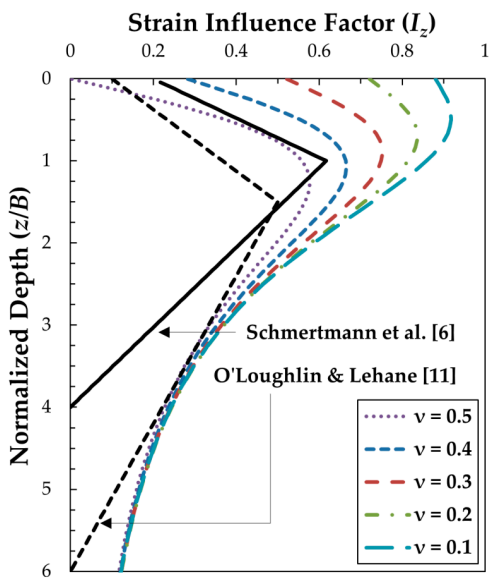

(b)

Figure 1. Strain influence factor for: (a) Square footing $(L / B=1.0)$; (b) Strip footing $(L / B \geq 10.0)$.

As mentioned before, Schmertmnann's method assumes linear elasticity of soil deformation. Similarly, $I_{z}$ used in Schmertmnann's method can be determined based on the elasticity theory after substituting the triangular $I_{z}$. In Figure 1, the strain influence factors derived from elasticity theory are shown. The unit vertical strains in cylindrical coordinates could be calculated from the constitutive relationship of Hooke's law:

$$
\varepsilon_{z}=\frac{1}{E}\left[\Delta \sigma_{z}-2 v \Delta \sigma_{r}\right]
$$

where $\Delta \sigma_{z}$ is the change in vertical stress, and $\Delta \sigma_{r}$ is the change in radial stress. The vertical stress increment on the axis passing through the center of a circular foundation is derived by integrating the Boussinesq's point loads over a distributed area:

$$
\Delta \sigma_{z}=q\left[1-\frac{1}{\left[1+(a / z)^{2}\right]^{1.5}}\right]
$$

where $q$ is applied stress on the foundation, $a$ is half of the foundation's diameter $(d / 2)$, and $z$ is the depth from the foundation base. Similarly, the expression for the radial stress increment is:

$$
\Delta \sigma_{r}=q\left[\frac{1}{2}+v-\frac{(1+v)}{\left[(a / z)^{2}+1\right]^{0.5}}+\frac{1}{2\left[(a / z)^{2}+1\right]^{1.5}}\right]
$$

The above equations can be applied to a circular foundation resting on an elastic, homogeneous, and an isotropic half-space medium. Thus, Young's modulus can be replaced with a constant such as 1 , and the vertical strain is determined by the difference between vertical and radial stresses in Equation (7). On the other hand, the $I_{z}$ addressed in Equation (1) can be calculated as the product of Young's modulus and the vertical strain (i.e., $I_{z}=E \varepsilon$ ) assuming a unit stress increment of 1 . Hence, the $I_{z}$ and vertical strain are identical under elasticity theory with $E$ of 1 , and $I_{z}$ equals the net stress increment in the vertical direction for a vertical deflection. In order words, the $I_{z}$ represents the ratio of the vertical stress distributed to the subsoil along the depth to the vertical stress applied at the surface. Using Equations (7)-(9) under unit stress $(q=1)$ and equivalent diameter, $d=(4 L B / \pi)^{0.5}$, for square or strip foundations, the strain influence factors are calculated and presented in Figure 1 for various Poisson ratios $(v)$. Compared with Schmertmann's triangle, the peak values from the elastic approach occur at shallower depths, and the values are much higher than those for Schmertmann's triangles, especially for low Poisson ratios $(v \leq 0.3)$, despite the peak values of triangles increasing with increasing applied pressure. This discrepancy could lead to significant errors in settlement prediction because low stiffness near the surface governs the deformation behavior. 
Schmertmann's triangles were derived by comparing deformation profiles beneath footings obtained from analysis using finite element method (FEM) and experiments. Poisson ratios used in the FEM study ranged from 0.3 to 0.48 . However, after recent developments in experimental apparatus and measurement techniques, sophisticated local strain measurement (excluding experimental errors) have become possible, and consequently it was reported that Poisson ratios less than 0.2 are adequate for all the soil types under the working load condition [18,19].

It seems that the abovementioned inaccuracies for Schmertmann's triangular $I_{z}$ occur not only for shallow depths (Poisson ratio) but also for deep depths (simplification), and hence the triangular $I_{z}$ can be improved for more accurate settlement calculation. In this study, feasibility of $I_{z}$ derived from the elasticity theory was verified and compared to the Schmertmann's triangular $I_{z}$.

\subsection{New Settlement Prediction Method}

A new settlement prediction method based on Schmertmann's approach is proposed combining the modulus derived from $V_{S}$ considering soil nonlinearity and $I_{z}$ from the elasticity theory as discussed in previous sections. The vertical stress level at each sublayer changes from the initial self-weight stress state, $\sigma_{\mathrm{ov}, j}$, to the stress condition upon additional footing load, which is equal to $\sigma_{\mathrm{o} v, j}+q_{i} I_{z, j}$. The change of stress condition causes increases in the small strain modulus, and this stress-dependent stress-strain relationship is presented in Equation (6). Following Lee \& Salgado [20], the deformation modulus, $E_{i j}$, considering both the stress-dependent stress-strain relationship and nonlinear stress-strain hyperbolic equation, can be expressed as:

$$
E_{i j}=E_{o, j}\left(\frac{\sigma_{o v, j}+q_{i} I_{z, j}}{\sigma_{o v, j}}\right)^{n}\left(1-f\left(\frac{q_{i} I_{z, j}}{\sigma_{\max }}\right)^{g}\right)
$$

where $\sigma_{\mathrm{ov}, j}$ is the initial vertical stress at depth $z_{j}, \sigma_{\max }$ is the ultimate bearing capacity, $E_{o, j}$ is the initial small-strain Young's modulus equal to $E_{0}=2 G_{0}(1+v)$. The solution from Meyerhof [21] can be applied to obtain the ultimate bearing capacity with respect to the shape of the footing. By combining Equations (1) and (10) and ignoring depth or time factors, the total settlement of each sublayer corresponding to the applied stress $q_{i}$ can be calculated using the following nonlinear stress-dependent modulus:

$$
s=q_{i} \sum \frac{I_{z i} \Delta z_{i}}{E_{0, i}\left(\frac{\sigma_{o v, i}+q I_{z, i}}{\sigma_{o v, i}}\right)^{n}\left(1-f\left(\frac{q I_{z, i}}{\sigma_{\max }}\right)^{g}\right)}
$$

Finally, the footing settlements are calculated based on Schmertmann's framework using $V_{S}$. For a foundation supported on granular soil, the elastic settlement is a major concern that needs consideration. In this study, the elastic load-settlement response of vertically loaded footings placed in granular soil was considered, while the plastic deformation of soil caused by the particle movement was not considered.

\section{Centrifuge Study}

In this study, three-dimensional centrifuge tests were performed to exam the load-settlement behavior of model footings embedded on sand deposits. Three model grounds with identical soil conditions were prepared for testing the model footings. The model footings with $L / B$ ratios of 1.0, 2.5, and 5.0, which represent square footing and rectangular footings, were placed on each of model ground.

\subsection{Experimental Description}

A large rigid box with a length, width, and height of 1000, 1000, and $477 \mathrm{~mm}$, respectively, was utilized as a centrifuge model box. Model footing with breadth equal to $60 \mathrm{~mm}$ was considered to avoid the arching effect caused by side wall friction of the model box. Figure 2 shows the model 
footings placed inside the model box. Stress distribution in the soil mass caused by the footing load was approximated using a simple approximate method (i.e., the 2:1 method), as presented by the dotted lines. The bottom areas estimated by the approximate method were expressed with respect to the $L / B$ ratio of the model footing as shown in Figure $2 b$. It is expected that when the breadth of the model footing is $60 \mathrm{~mm}$, the deformation behavior would not be affected by the wall friction of the model box, even in the case where the $L / B$ ratio equals 5.0.

Dry silica sand was used for model ground construction and its basic properties are presented in Table 1. Before constructing the model ground, bender elements (BE) were installed in the model box for the measurement of the $V_{S}$ of model ground while accelerating the model in a centrifuge [22]. A total of 15 pairs of bender elements arrays were installed on aluminum rods and the rods were attached on the bottom of the model box, as presented in Figure 2a. The bender elements were equipped vertically on the rods to generate and measure $V_{S, H H}$, for which directions of propagating and particle motion are horizontal. Source bender elements were made in parallel, whereas receiver bender elements were made in series to enhance sending and receiving of the shear wave. The bender element arrays cover depths up to $5 B$ beneath the model footing, and the tip-to-tip distance between source and receiver bender elements was $300 \mathrm{~mm}$. This distance of $300 \mathrm{~mm}$ between the bender pair minimizes the effect of the supporting rods and bender elements on the soil mass beneath footings. The soil mass is not affected by these anomalies up to a depth of $4 B$ based on the 2 to 1 method, as presented in Figure 2a. After installing the rods, the model ground was constructed by a dry compaction method, and this procedure was repeated 3 times for each test. The final relative density (Dr) of the prepared model ground was $85 \%$, and the height of the prepared soil model was $460 \mathrm{~mm}$, which is 7 times larger than the footing breadth.

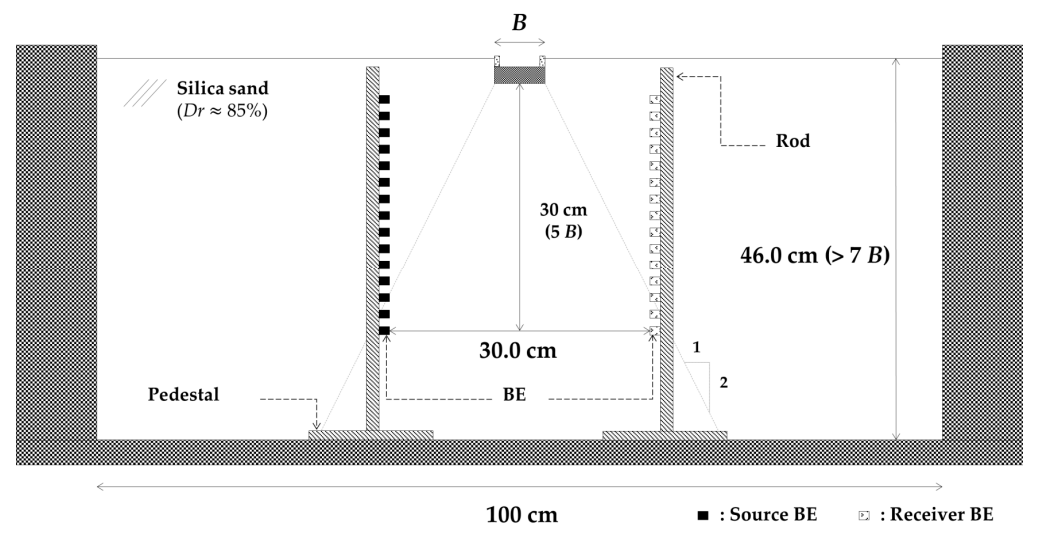

(a)

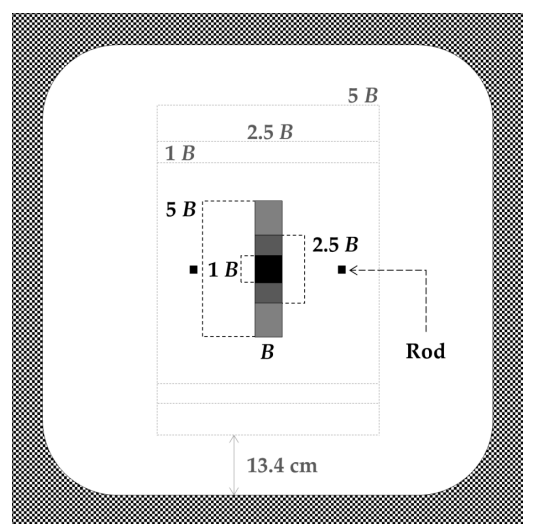

(b)

Figure 2. Model configuration: (a) Cross Section; (b) Top View. 
Table 1. Physical properties of the silica sand.

\begin{tabular}{cc}
\hline Properties & Silica Sand \\
\hline Unified soil classification (USCS) & SP \\
Median particle size $\left(D_{50}\right), \mathrm{mm}$ & 0.22 \\
Curvature coefficient $\left(C_{C}\right)$ & 1.11 \\
Uniformity coefficient $\left(C_{U}\right)$ & 1.96 \\
Specific gravity $\left(G_{S}\right)$ & 2.65 \\
Plasticity index $(P I)$ & $\mathrm{NP}$ \\
Maximum void ratio $\left(e_{\max }\right)$ & 1.130 \\
Minimum void ratio $\left(e_{\min }\right)$ & 0.611 \\
\hline
\end{tabular}

The model footings were made of aluminum having same width and height of $60 \times 20 \mathrm{~mm}$ for the three different models. The length of the model footings was $60 \mathrm{~mm}, 150 \mathrm{~mm}$, and $300 \mathrm{~mm}$ for $L / B$ ratios of 1.0, 2.5, and 5.0, respectively. The model footings were placed at a height of $430 \mathrm{~mm}$ from the bottom of the soil deposit, and they were laid in a perpendicular direction to the shear wave ray path, as presented in Figure 2b. In order to minimize the bedding error during vertical loading, model footings were embedded into the soil. An embedding depth of $30 \mathrm{~mm}$ was used for considering the equivalent soil weight with model footing to remove the overburden pressure before vertical loading. Acryl resin was attached on the top of model footing's perimeter to prevent the collapse of soil into the footing, as shown in Figure 2a.

The experiments were conducted in a $5 \mathrm{~m}$ platform radius geotechnical centrifuge having a $240 \mathrm{~g}$-ton capacity [23]. A target centrifugal acceleration level of $40 \mathrm{~g}$ was adopted, and thus model footings with a prototype length of $2.4 \mathrm{~m}, 6.0 \mathrm{~m}$, and $12.0 \mathrm{~m}$ were simulated according to the centrifuge scaling law. Vertical loading equipment was used to apply a vertical load, and the loading condition was controlled with a constant velocity of $0.075 \mathrm{~mm} / \mathrm{s}$. After the 1 st loading, the load was completely removed, and the 2nd loading was then applied with a larger load than that of the 1st loading. The vertical load and the settlement of the foundation were measured by load cell and the linear variable differential transformer (LVDT), respectively. Before the 1st and 2nd loadings, $V_{S}$ was measured using the embedded bender elements. A $100 \mathrm{~Hz}$ square signal was utilized as an input signal. The shear wave signals were detected by a receiver in cross-hole type configuration and stacked 32 times to increase the signal to noise ratio.

\subsection{Results and Discussion}

\subsubsection{Shear Wave Velocity $\left(V_{S}\right)$}

The $V_{S}$ was calculated after determining the first arrival time of received shear wave signals and the tip-to-tip distance between the two corresponding bender elements [24]. Interpolation based on a regression analysis was conducted to produce continuous $V_{S}$ profiles for precise predictions because the shear wave was detected by point-based measurements. The measured $V_{S}$ values and theoretical confining stress were used for the regression analysis as shown below [25]:

$$
V_{s}=C_{s}\left(\sigma^{\prime} / P_{a}\right)^{n / 2}
$$

where $C_{S}$ is material constant, $\sigma^{\prime}$ is effective confining stress, and $P_{a}$ is atmosphere pressure to normalize the stress term, which was generally assumed to be $100 \mathrm{kPa}$. In this study, the theoretically calculated vertical effective stress was adopted as the effective confining stress in Equation (12).

Figure 3 shows the measured and interpolated $V_{S}$ profiles with respect to depth, which is normalized by footing breadth $(B)$ for the three experiments. As shown in the figure, three $V_{S}$ profiles before the 1st loading are almost identical, which means the model grounds were constructed homogeneously and uniformly. It is interesting to note that the $V_{S}$ measured before 2nd loading becomes greater than the 1st loading and the depth at which $V_{S}$ increased was deeper with increasing 
$L / B$ ratio. This could be attributed to residual horizontal stress in the subsurface after the 1st loading, and the area influenced by the residual stress deepened with increasing $L / B$ ratio. Given that the residual stress is evidence of load transfer during the 1st loading, the residual stress can be correlated with the $I_{z}$. The $I_{z}$ profiles from Schmertmann et al. [6] and elasticity theory reflect the aforementioned trend that the stress increment transmitted from the surface is deeper with increasing $L / B$ ratio. The influenced depth depending on the $L / B$ ratio converges to zero at $2.0 B$ depth for square footing and at $4.0 B$ depth for strip footing as shown in Figure 1. Figure 4 shows the discrepancies between the $V_{S}$ measured before 2nd loading and that before 1st loading with respect to depth, which is normalized by footing breadth $(B)$. In this figure, it is possible to confirm the phenomena mentioned before more clearly. The influenced depth and depth to peak, which indicates the maximum $V_{S}$ difference, increase with increasing $L / B$ ratio as presented by shading.

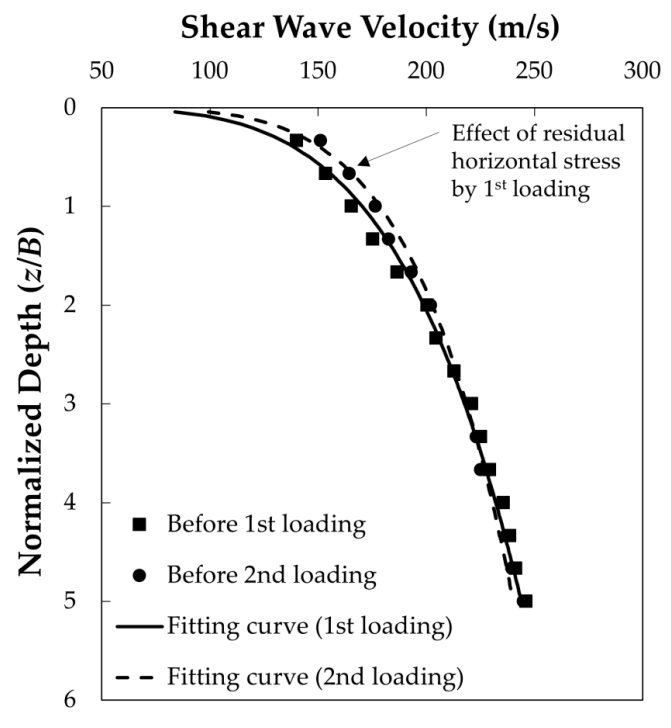

(a)

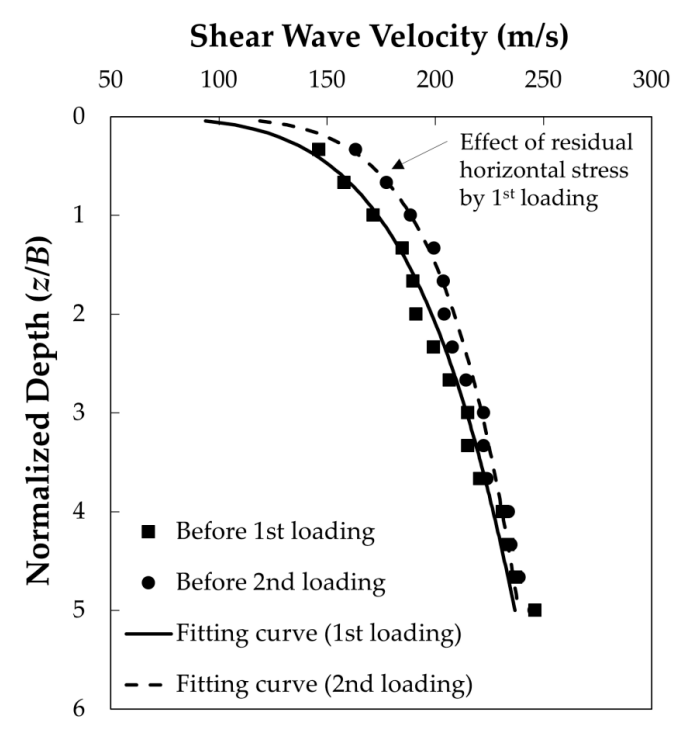

(b)

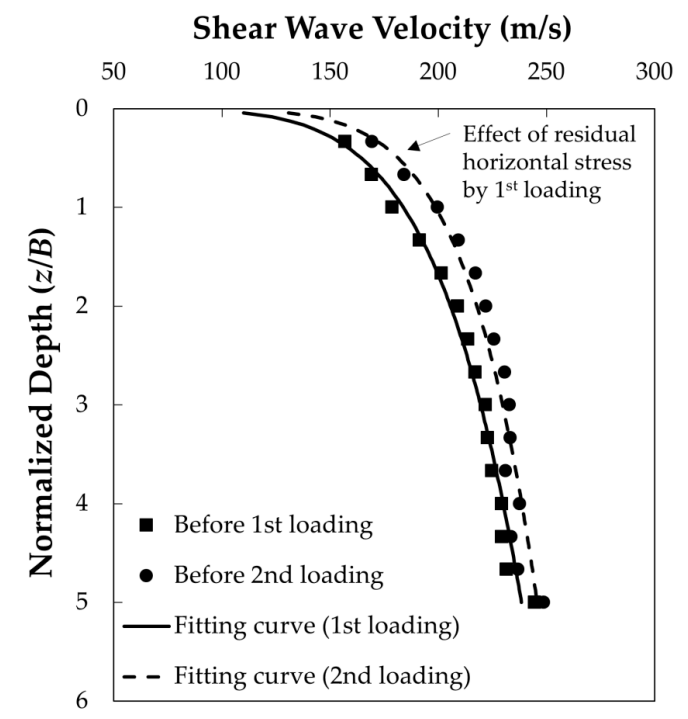

(c)

Figure 3. Shear wave velocity profiles: (a) $L / B=1.0 ;$ (b) $L / B=2.5 ;$ (c) $L / B=5.0$. 


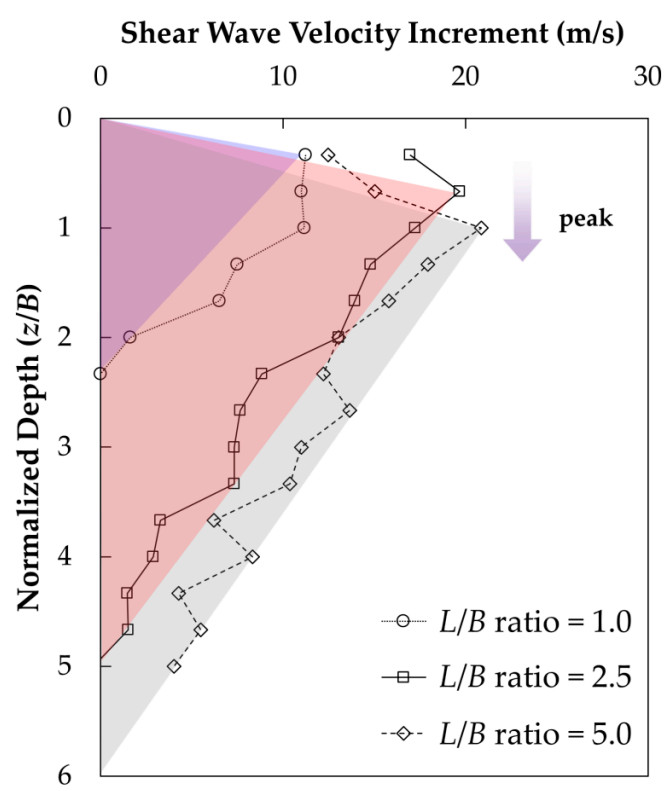

Figure 4. Shear wave velocity increments after 1st loading.

\subsubsection{Soil Parameters Used in Predictions}

In order to compare the load-settlement curves from centrifuge tests with predictions, two different $I_{z}$ values from the elasticity theory and Schmertmann's triangles [6] were utilized simultaneously. In case of the elastic solution, it is necessary to determine the Poisson ratio. As discussed in the previous section, it is evident that the Poisson ratio is less than typically imagined and its range under the working load condition is less than $0.2[18,19]$. A Poisson ratio of 0.2 was, therefore, selected as an equivalent value, and the corresponding shapes with Schmertmann's triangles are shown in Figure 5. As the peak value of Schmertmann's triangle increases with increasing surcharge load $(q)$, the shapes shown in Figure 5 are fixed when the surcharge load is $300 \mathrm{kPa}$ for comparison.

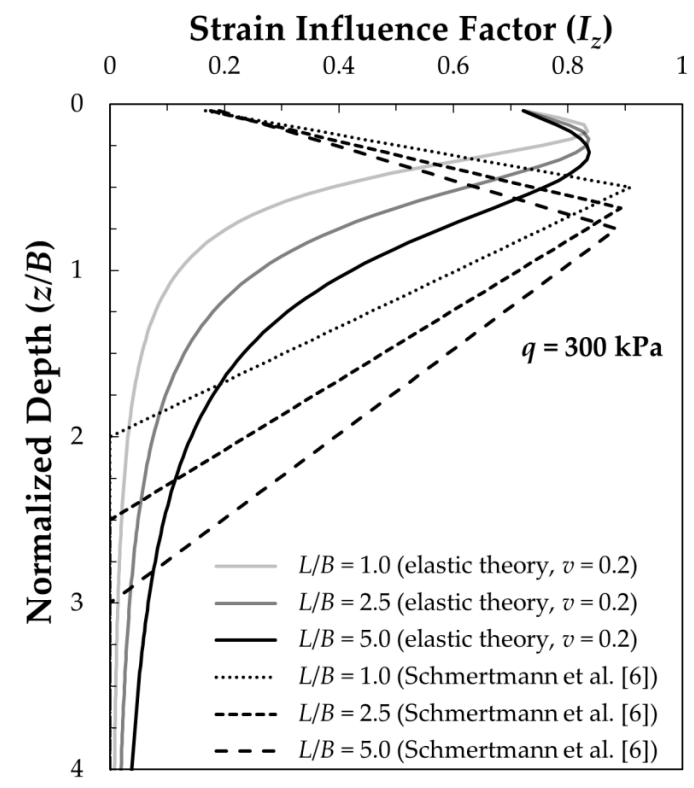

Figure 5. Strain influence factor $\left(I_{z}\right)$ profiles used in predictions. 


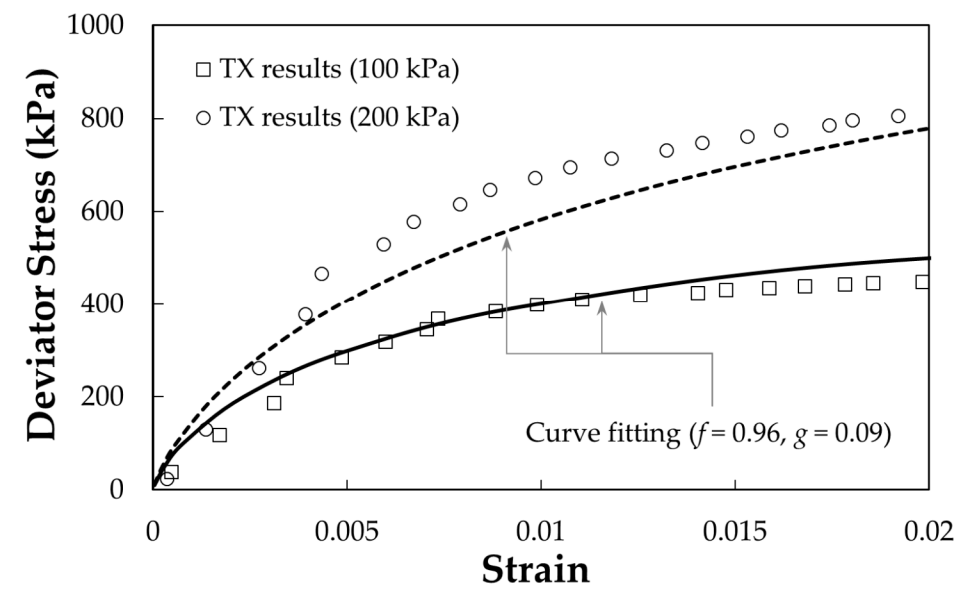

Figure 6. Determination of model parameters $f$ and $g$ from triaxial test (TX) tests.

Table 2. Soil parameters for settlement predictions.

\begin{tabular}{cccccc}
\hline$f$ & $g$ & $n$ & Poisson Ratio, $\boldsymbol{v}$ & Friction Angle, $\boldsymbol{\varnothing}$ & Bearing Capacity, $\sigma_{\max }$ \\
\hline \multirow{2}{*}{0.96} & 0.09 & 0.5 & \multirow{2}{*}{0.2} & $43^{\circ}$ & $\begin{array}{l}L / B=1.0,3439 \mathrm{kPa} \\
L / B=2.5,2729 \mathrm{kPa} \\
\end{array}$ \\
& & & & $L / B=5.0,2506 \mathrm{kPa}$ \\
\hline
\end{tabular}

Given that the nonlinear deformation characteristic of soils is determined by certain parameters in Equation (11), the selection of appropriate soil parameters is important. For the stress exponent, $n$, 0.5 was used since this value is commonly assumed for clean quartz and silica sands $[15,20,26]$. For the determination of model parameters, $f$ of 0.96 and $g$ of 0.09 were determined through regression analysis using the results of the triaxial test (TX) with the confining pressure of $100 \mathrm{kPa}$ and $200 \mathrm{kPa}$ as shown in Figure 6. More weight was taken into account for the results from the small-strain region. The ultimate bearing capacity, $\sigma_{\max }$, depends on the $L / B$ ratio and can be obtained using the Meyerhof method [21] based on the internal friction angle. All soil parameters used in predictions are tabulated in Table 2. In order to determine the initial stiffness of model ground, the $V_{S}$ profile measured before each loading was used in each settlement prediction for representing the reasonable stress state of model ground. Finally, a thickness of each sublayer $\left(\Delta z_{i}\right)$ of $0.1 \mathrm{~m}$ was applied in Equation (11).

\subsubsection{Comparisons of Load-Settlement Curves with Predictions}

In Figures 7-9, measured model settlements were compared with the predictions for $L / B$ ratios of $1.0,2.5$, and 5.0, respectively. Settlement of model footing was normalized using the footing breadth $(B)$, and the load-settlement curves were presented up to the normalized settlement of $3 \%$. To take into account the foundation load eccentricity, load inclination following step-by-step procedures suggested by Briaud [27] was adopted as shown in Figure 7. The straight line part of the 2nd load-settlement curve was extended to zero pressure, thereby shifting the vertical axis to the value of $s / B$, where that straight line portion intersects the horizontal axis.

The centrifuge test results for 1st loading in Figures 7-9 showed very soft deformable behavior compared with the two predictions, regardless of the $L / B$ ratio. It could be reasoned that particle rearrangement, caused by interparticle slip and rotation, governs the deformation behavior rather than the compression of the soil mass related to the modulus for the 1st loading [28]. Irrecoverable plastic behavior caused by the particle rearrangement started at very small strain region and, then, the elastoplastic behavior of soil emerged after a very small elastic limit. Since modeling of soil behavior utilizing the stiffness is valid until the elastic limit, it is not possible that the developed settlement prediction method using the hyperbolic stress-strain model in this study estimates the soil deformation behavior under the normally-consolidated (NC) stress state governed by relative particle movements. 
The elastoplastic behavior of soil after the elastic limit (i.e., yield point) can be considered by the plasticity theory; however, this kind of complicated modeling technique is not suitable for settlement prediction in engineering practice using a simple tool such as Schmertmann's method.

On the contrary, the centrifuge test results and two predictions for the 2nd loading show different tendencies. Unlike the results from the 1st loading, the predictions for the three cases using $I_{z}$ from elasticity theory yielded good agreement with centrifuge test results, especially up to the settlements corresponding to the maximum previous loadings (pre-consolidation pressure). Because the yield points of the three model grounds increased due to the maximum previous loading of the 1st loading cycle, the elastic limit also increased more than that of the original NC stress state ground. Major particle rearrangement occurred and disappeared considerably during the 1st loading cycle, and this particle rearrangement might readily induce more compact configuration of structures. Consequently, elastic soil deformation, which is represented by the modulus, was generated in the recompression stage i.e., 2nd loading. As mentioned before, modeling of soil deformation behavior using a nonlinear hyperbolic stress-strain model based on stiffness is possible up to the elastic limit, and thus the developed settlement prediction method correctly evaluates the load-settlement behavior in the enlarged elastic region.

After the maximum previous loading in 2nd loading stage, predictions using $I_{z}$ from elasticity theory underestimate the settlements of model footing. As the plastic deformation of soil was not considered in the developed settlement prediction method, the predictions and load-settlement curves from centrifuge tests showed greater discrepancies, like in the case of the 1st loading stage. On the other hand, predictions using $I_{z}$ from Schmertmann et al. [6] gave similar global load-settlement behavior with the centrifuge test results up to the normalized settlement of $3 \%$ even though there were still slight differences before and after the maximum previous loading. Given that the settlement prediction method developed by Schmertmann et al. [6] is a semi-empirical method based on a combination of elasticity theory, laboratory tests and numerical analysis, there is a possibility that Schmertmann's varying triangular $I_{z}$ dependent on applied pressure had been developed to consider the nonlinear deformation behavior of soil over the elastic limit empirically for simple use in engineering, rather than using the relatively complicated plasticity theory. Therefore, the use of the $I_{z}$ from Schmertmann et al. [6] generally results in an approximate solution throughout the entire strain region despite the fact that the modulus was converted from $V_{S}$ not $q_{c}$ in this study.

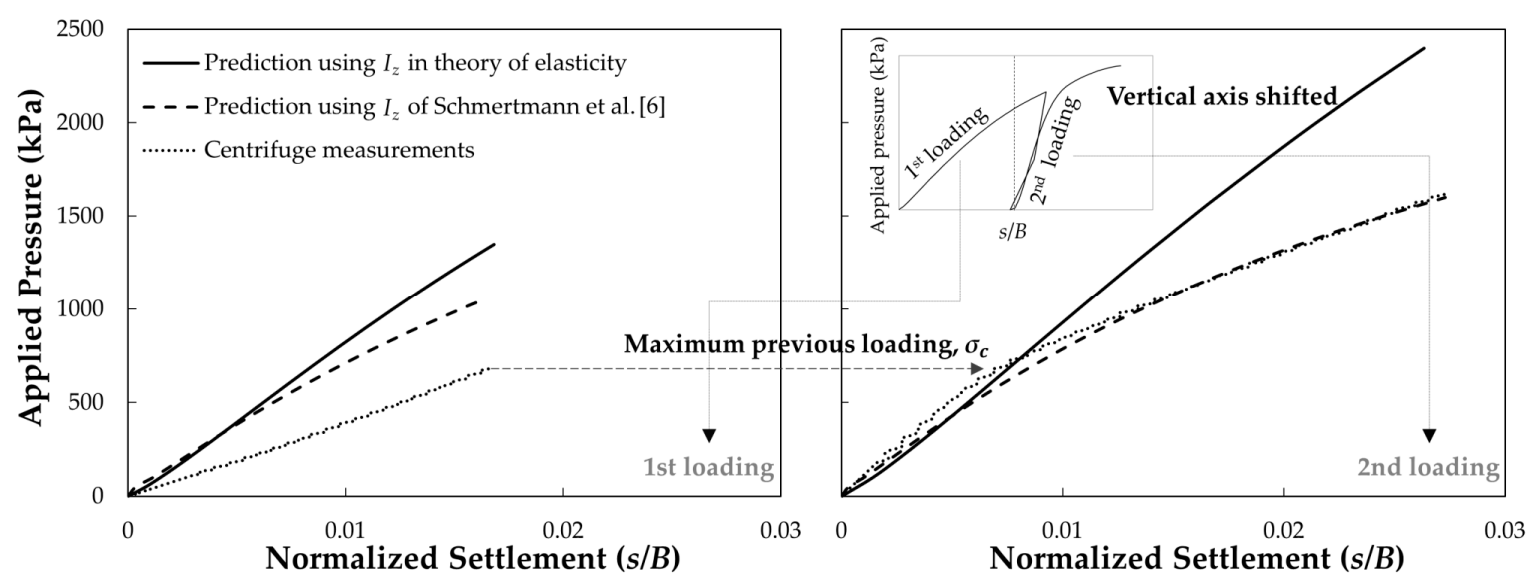

(a)

(b)

Figure 7. Comparisons of load-settlement curves with predictions $(L / B=1.0)$ : (a) 1st loading; (b) 2nd loading. 


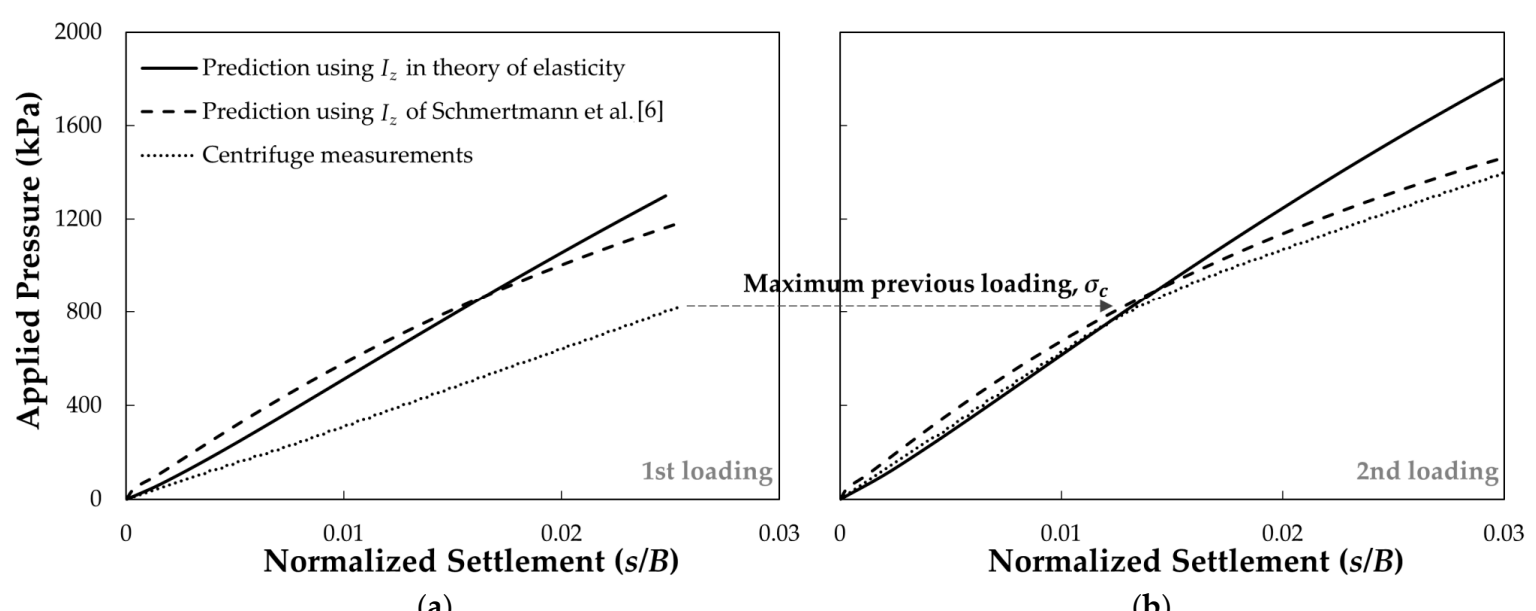

(a)

(b)

Figure 8. Comparisons of load-settlement curves with predictions $(L / B=2.5)$ : (a) 1st loading; (b) 2nd loading.

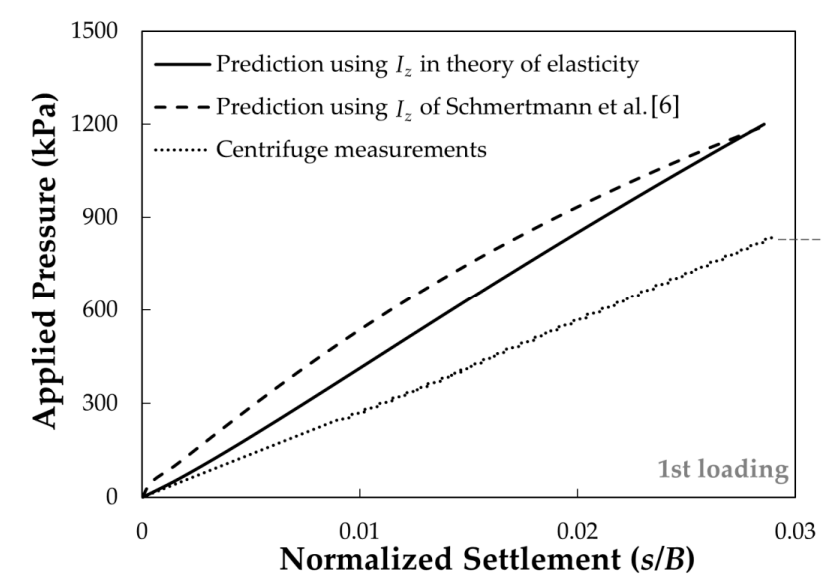

(a)

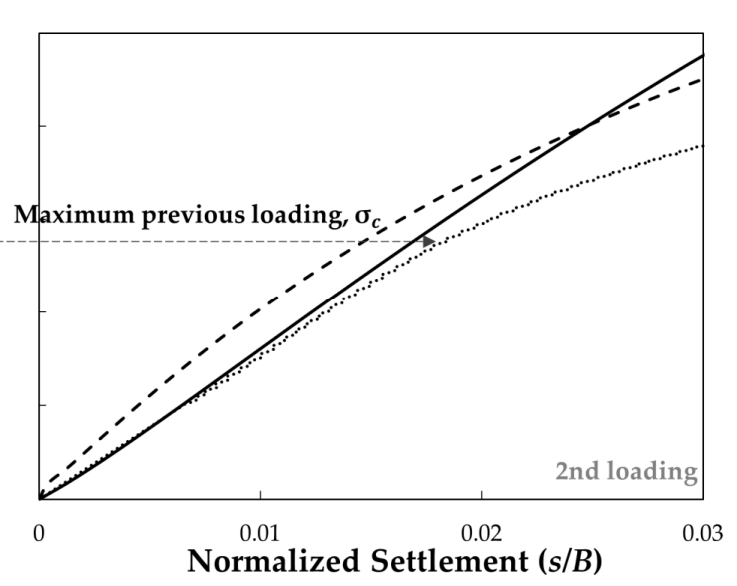

(b)

Figure 9. Comparisons of load-settlement curves with predictions $(L / B=5.0)$ : (a) 1st loading; (b) 2nd loading.

\subsubsection{Modulus Profiles}

In order to check modulus profiles utilized in settlement predictions, numerically computed modulus profiles mobilized during the settlement predictions are shown with respect to normalized depth $(z / B)$ in Figure 10. Each modulus profile was obtained at each applied pressure $(q)$ from $100 \mathrm{kPa}$ to $1600 \mathrm{kPa}$, and the normalized settlements $(s / B)$, corresponding to the applied pressure are presented as a percentage. For determination of initial modulus profile, the shear wave velocity profiles measured before the 2 nd loading of each $L / B$ ratio were utilized. General trends in the modulus profile transitions were observed such that the modulus increased under low applied pressures and decreased near the surface above $800 \mathrm{kPa}$. The threshold depth, where the modulus becomes smaller than the previous value, deepened with increasing $L / B$ ratio. This phenomenon could occur due to the shapes of the $I_{z}$ derived from the elasticity theory. To sum up, the increase in modulus by confining pressure and modulus reduction by strain level are properly modelled in the developed settlement prediction method considering the shape of $I_{z}$. 


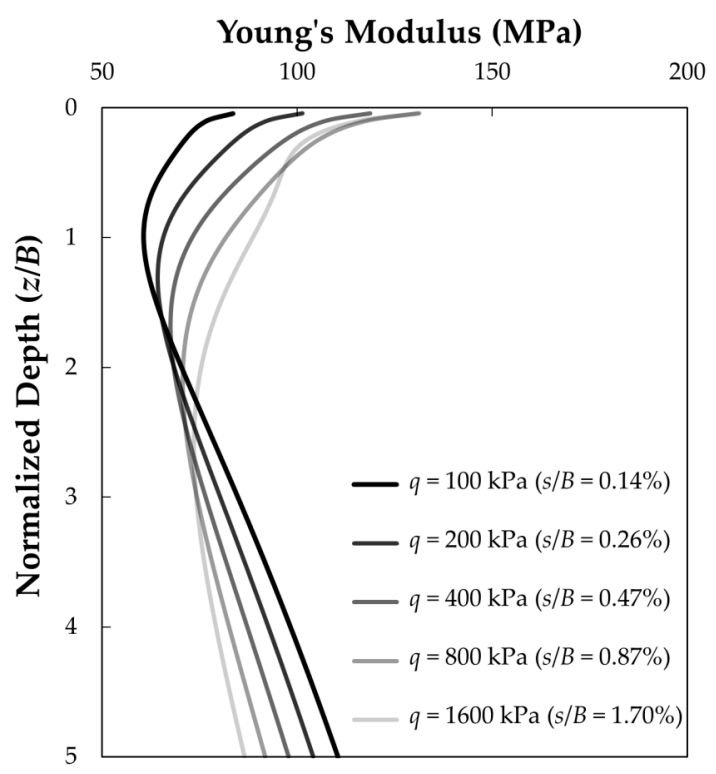

(a)

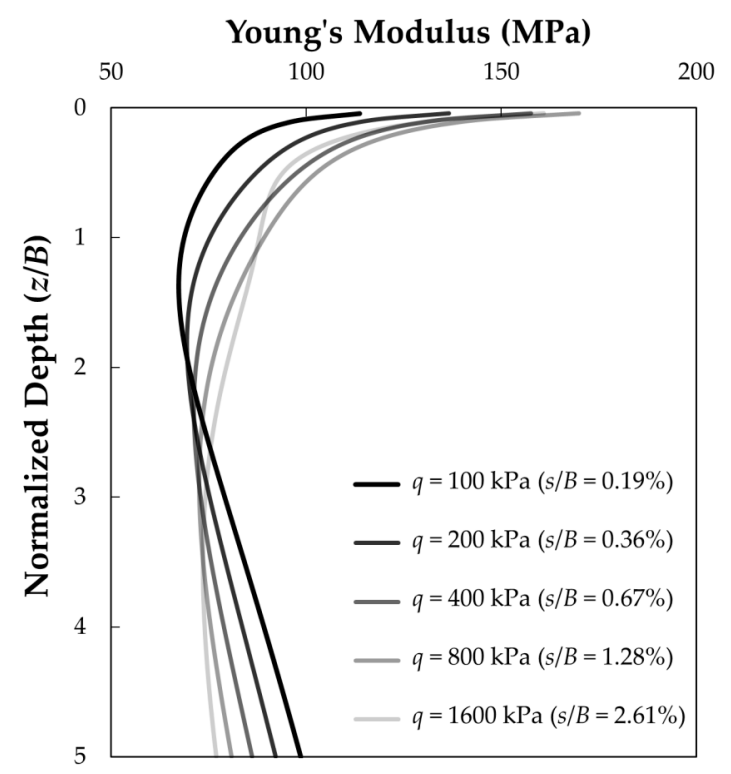

(b)

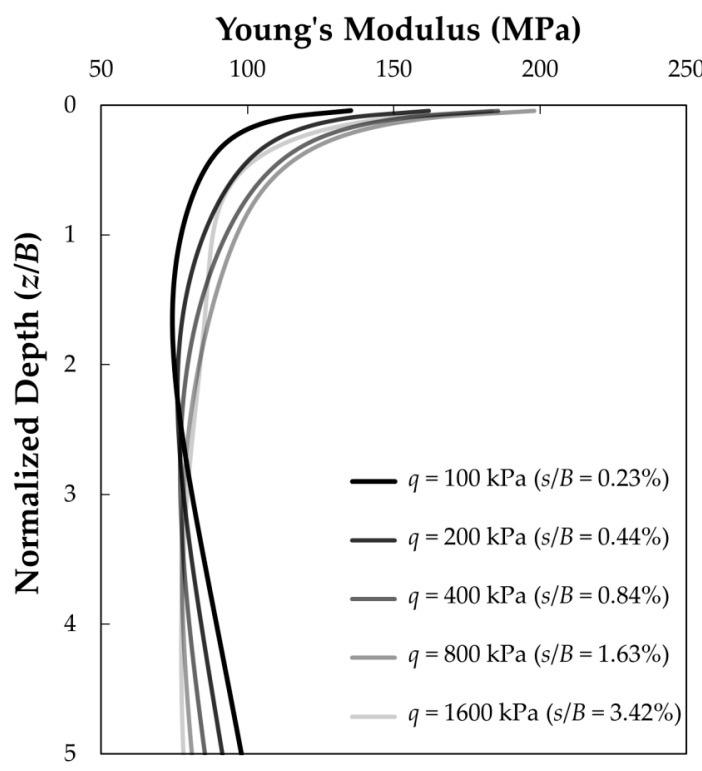

(c)

Figure 10. Mobilized Young's modulus profiles: (a) $L / B=1.0 ;$ (b) $L / B=2.5 ;$ (c) $L / B=5.0$.

\section{Discussion}

It is confirmed that the developed settlement prediction method adequately predicts the settlement of footings on over-consolidated (OC) deposits, where particle movement is minimal, based on centrifuge tests. On the other hand, there is a certain limitation of the developed method for normally-consolidated (NC) granular soils even though most sites where the construction of the shallow foundation is suitable have slightly- or heavily-consolidated ground conditions with substantial bearing capacity. To solve this problem, Burland \& Burbidge [4] suggested a settlement prediction method and considered the soil compressibility differently from the SPT- $N$ value for NC and OC deposits using empirical coefficients. Leonards \& Frost [29] also proposed a settlement prediction method based on Schmertmann's conceptual framework. In the method, the settlement or the soil stiffness is adjusted by adopting the ratio of the stress increment corresponding to the NC portion and the OC portion in a given layer to the total increment of stress in the layer. Consequently, the empirical 
coefficient considering the NC and OC stress condition based on pre-consolidation pressure has to be adopted in the developed method in further studies.

\section{Conclusions}

A new settlement prediction method was developed and discussed in this study. Complete load-settlement behavior of footing could be predicted using $V_{S}$-based Schmertmann's framework considering soil non-linearity. The main findings from this study are summarized as follows:

(i) For the reliable stiffness estimation of soil layers, the modulus variation by confinement stress and strain level increment was reflected in the developed method based on the $V_{S}$. The vertical stress increment with depth can be determined from the $I_{z}$ calculated from the elasticity theory. The settlements of each sublayer are calculated based on the modulus and stress determined at each depth, and then added to give total settlements.

(ii) The prediction using the $I_{z}$ based on elasticity theory showed reasonable agreement with the centrifuge test results for 2 nd loading stages due to an enlarged elastic limit. The modulus profile mobilized in the predictions showed that the modulus variation by confining pressure and strain level is properly modeled in the developed settlement prediction method considering the shape of $I_{z}$.

(iii) The centrifuge results for the 1st loading (NC condition) showed very soft deformable behavior compared with the two predictions regardless of the $L / B$ ratio. It could be concluded that the particle rearrangement such as interparticle slip and rotation governs the deformation behavior rather than the compression of the soil mass related to the soil modulus. To take into account the normally-consolidated (NC) stress state governed by relative particle movements, the empirical coefficient needs to be adopted in the developed method.

Acknowledgments: This research was supported by Basic Science Research Program through the National Research Foundation of Korea (NRF) funded by the Ministry of Education (grant number 2014R1A6A3A04056405). Also, support from Basic Research Project of Korea Institute of Geoscience and Mineral Resources (KIGAM) is acknowledged.

Author Contributions: Hyung Ik CHO organized the paperwork, performed the centrifuge tests and completed the development of the new settlement prediction method; Nam Ryong KIM initially suggested the idea, and helped with the centrifuge tests; Heon Joon PARK processed the data collected from the centrifuge tests and interpreted the data; Dong Soo KIM supervised the research activities and provided guidance and suggestions for the proposed method; all authors contributed to the writing of each part.

Conflicts of Interest: The authors declare no conflict of interest.

\section{References}

1. Kramer, S.L. Performance-based design methodologies for geotechnical earthquake engineering. Bull. Earthq. Eng. 2014, 12, 1049-1070. [CrossRef]

2. Meyerhof, G.G. Shallow foundations. J. Soil Mech. Found. Div. 1965, 91, 21-31.

3. Peck, R.B.; Bazaraa, A.R.S.S. Discussion of paper by D'Appolonia et al. J. Soil Mech. Found. Div. 1969, 96, 305-309.

4. Burland, J.B.; Burbidge, E. Settlement of foundations on sand and gravel. Proc. Inst. Civ. Eng. 1985, 76, 1325-1381. [CrossRef]

5. Schmertmann, J.H. Static cone to compute static settlement over sand. J. Soil Mech. Found. Div. 1970, 96, 1011-1043.

6. Schmertmann, J.H.; Brown, P.R.; Hartman, J.P. Improved strain influence factor diagrams. J. Geotech. Eng. Div. 1978, 104, 1131-1135.

7. Berardi, R.; Jamiolkowski, M.; Lancellotta, R. Settlement of shallow foundations in sands selection of stiffness on the basis of penetration resistance. In Proceedings of the Geotechnical Engineering Congress, Boulder, CO, USA, 10-12 June 1991. 
8. Lehane, B.M.; Doherty, J.P.; Schneider, J.A. Settlement prediction for footings on sand. Deform. Charact. Geomater. 2008, 1, 133-150.

9. Lee, J.H.; Salgado, R. Estimation of footing settlement in sand. Int. J. Geomech. 2002, 2, 1-28. [CrossRef]

10. Lee, J.H.; Eun, J.; Prezzi, M.; Salgado, R. Strain influence diagrams for settlement estimation of both isolated and multiple footings in sand. J. Geotech. Geoenviron. Eng. 2008, 134, 417-427. [CrossRef]

11. O'Loughlin, C.D.; Lehane, B.M. Nonlinear Cone Penetration Test-Based Method for Predicting Footing Settlements on Sand. J. Geotech. Geoenviron. Eng. 2009, 136, 409-416. [CrossRef]

12. Kim, D.S.; Park, H.J.; Cho, H.I. Measurement and Application of Shear Wave Velocity to Various Geotechnical Problems. In Proceedings of the 6th International Symposium on Deformation Characteristics of Geomaterials, IS-Buenos Aires 2015, Buenos Aires, Argentina, 15-18 November 2015.

13. Fahey, M. Shear modulus of cohesionless soil: Variation with stress and strain level. Can. Geotech. J. 1992, 29, 157-161. [CrossRef]

14. Fahey, M.; Carter, J.P. A finite element study of the pressuremeter test in sand using a nonlinear elastic plastic model. Can. Geotech. J. 1993, 30, 348-362. [CrossRef]

15. Fahey, M.; Robertson, P.K.; Soliman, A.A. Towards a rational method of predicting settlements of spread footings on sand. In Proceedings of the Vertical and Horizontal Deformations of Foundations and Embankments, College Station, TX, USA, 16-18 June 1994.

16. Lehane, B.; Fahey, M. A simplified nonlinear settlement prediction model for foundations on sand. Can. Geotech. J. 2002, 39, 293-303. [CrossRef]

17. Mayne, P.W.; Poulos, H.G. Approximate displacement influence factors for elastic shallow foundations. J. Geotech. Geoenviron. Eng. 1999, 125, 453-460. [CrossRef]

18. Tatsuoka, F.; Teachavorasinskun, S.; Dong, J.; Kohata, Y.; Sato, T. Importance of measuring local strains in cyclic triaxial tests on granular materials. ASTM Spec. Tech. Pub. 1994, 1213, 288.

19. Lo Presti, D.C.F.; Pallara, O.; Puci, I. A modified commercial triaxial testing system for small strain measurements: Preliminary results on Pisa clay. Geotech. Test. J. 1995, 18, 15-31.

20. Lee, J.H.; Salgado, R. Determination of pile base resistance in sands. J. Geotech. Geoenviron. Eng. 1999, 125, 673-683. [CrossRef]

21. Meyerhof, G.G. Some recent research on the bearing capacity of foundations. Can. Geotech. J. 1963, 1, 16-26. [CrossRef]

22. Kim, N.R.; Kim, D.S. A shear wave velocity tomography system for geotechnical centrifuge testing. Geotech. Test. J. 2010, 33, 434-444.

23. Kim, D.S.; Kim, N.R.; Choo, Y.W.; Cho, G.C. A newly developed state-of-the-art geotechnical centrifuge in Korea. KSCE J. Civ. Eng. 2013, 17, 77-84. [CrossRef]

24. Youn, J.U.; Choo, Y.W.; Kim, D.S. Measurement of small-strain shear modulus $G_{\max }$ of dry and saturated sands by bender element, resonant column, and torsional shear tests. Can. Geotech. J. 2008, 45, 1426-1438. [CrossRef]

25. Cho, H.I.; Park, H.J.; Kim, D.S.; Choo, Y.W. Evaluation of $\mathrm{K}_{\mathrm{o}}$ in Centrifuge Model using Shear Wave Velocity. Geotech. Test. J. 2014, 37, 255-267. [CrossRef]

26. Yu, P.; Richart, F.E., Jr. Stress ratio effects on shear modulus of dry sands. J. Geotech. Eng. 1984, 110, 331-345. [CrossRef]

27. Briaud, J.L. Spread footings in sand: Load settlement curve approach. J. Geotech. Geoenviron. Eng. 2007, 133, 905-920. [CrossRef]

28. Mesri, G.; Vardhanabhuti, B. Compression of granular materials. Can. Geotech. J. 2009, 46, 369-392. [CrossRef]

29. Leonards, G.A.; Frost, J.D. Settlement of shallow foundations on granular soils. J. Geotech. Eng. 1988, 114, 791-809. [CrossRef]

(C) 2017 by the authors. Licensee MDPI, Basel, Switzerland. This article is an open access article distributed under the terms and conditions of the Creative Commons Attribution (CC BY) license (http:/ / creativecommons.org/licenses/by/4.0/). 